\title{
Distributed Mobility Tracking for Ad Hoc Networks Based on an Autoregressive Model
}

\author{
Zainab R. Zaidi and Brian L. Mark \\ Dept. of Electrical and Computer Eng. \\ George Mason University \\ Fairfax, VA 22030, U.S.A.
}

\begin{abstract}
In ad hoc networks, node mobility causes the network topology to change dynamically over time, which complicates important tasks such as routing and flow control. We propose a distributed scheme for accurately and efficiently tracking the mobility of nodes in ad hoc networks. A first-order autoregressive model is used to represent the evolution of the mobility state of each node, which consists of position, velocity, and acceleration. Each node uses an extended Kalman filter to estimate its mobility state by incorporating network-based signal measurements and the position estimates of the neighbor nodes. Neighbor nodes exchange their position estimates periodically by means of HELLO packets. Each node re-estimates its mobility model parameters, allowing the scheme to adapt to changing mobility characteristics. In practice, a small number of reference nodes with known coordinates is required for accurate mobility tracking. Simulation results validate the accuracy of the proposed tracking scheme.
\end{abstract}

\section{Introduction}

The absence of a fixed infrastructure in mobile ad hoc networks makes them suitable for applications such as military battlefields, disaster relief, emergency situations, and low cost commercial communication systems. However, the flexibility of a highly dynamic ad hoc network complicates important network control and management tasks such as routing, flow control, and power management. The mobility of nodes leads to dynamic changes in link availability, resulting in frequent route failures. This can adversely affect network performance in terms of increased packet loss or delay. If the dynamics of the network topology could be predicted in advance, a route discovery mechanism could select paths that were more stable or long-lived in order to avoid or reduce route failures. In ad hoc networks, the network topology dynamics can be inferred from the mobility of

\footnotetext{
* This work was supported by the U.S. National Science Foundation under Grant No. ACI-0133390 and Grant No. CCR-0209049, as well as by a grant from the Cleveland Foundation.
} 
the nodes. Therefore, a mechanism to track node mobility could be of significant benefit to resource management in ad hoc networks.

We present a distributed scheme to accurately track real-time node mobility in an ad hoc network in terms of an autoregressive model (AR-1) of mobility [1]. The AR-1 model is sufficiently simple to enable real-time mobility tracking, but general enough to accurately capture the characteristics of realistic mobility patterns in wireless networks. Some of the more prominent mobility models (cf. [2]) that have been proposed in the literature include random walk models, the random waypoint model, Gauss-Markov models, and linear system models. The random walk and random waypoint models have the important feature of simplicity, making them amenable for use in simulations and in some cases analytical modeling of wireless network behavior. However, recent work has shown that such models do not accurately represent actual user trajectories in real wireless networks [3]. Consequently, such models may result in misleading characterizations of network performance. Moreover, such models are not sufficiently rich to enable accurate and precise real-time mobility tracking.

A linear system model of mobility can capture the characteristics of realistic mobile trajectories and has been applied to real-time mobility tracking for cellular and ad hoc networks $[4,5]$. However, the specification of an optimal set of linear system model parameters is not straightforward. Mobility tracking schemes derived from the linear dynamic system model perform well as long as the model parameters match the mobility characteristics of the user. However, they cannot adapt to significant changes in the model parameters over time. The AR-1 model has a similar structure to the linear system model. An important feature of the AR-1 model is that optimal parameter estimates for the model can be determined efficiently via the Yule-Walker equations [6].

Our proposed mobility estimation scheme integrates optimal parameter estimation via the Yule-Walker equations with mobility state estimation using Kalman filtering. The mobility state estimation scheme utilizes network-based signal measurements, such as received signal strength indicators (RSSI) or timeof-arrival of signals (TOA), to infer relative distances between neighbor nodes. A key feature of the proposed tracking scheme is that it provides estimates of position, velocity, and acceleration for each node. The algorithm is distributed and computationally feasible for real-time tracking applications, as it requires that each node perform a constant number of Kalman filtering and Yule-Walker steps at each estimation instant. Further, the mobility information generated by the tracking scheme can be used to predict future mobility behavior and hence future link availability in an ad hoc network.

The integrated mobility estimation scheme can adapt to changes in the mobility characteristics over time, since the model parameters are continuously re-estimated using new observation data. Moreover, the tracking scheme incurs relatively low communication overhead, which consists of periodic broadcasts of short HELLO messages containing mobility state information. Each node executes a Kalman filter to track its mobility state based on the observation data received from neighbor nodes. The accuracy of the tracking scheme improves as 
the nodal density increases. The presence of a small number of reference nodes with known coordinates in the network is needed to maintain accurate mobility tracking performance.

Several location estimation algorithms have been proposed in the literature for ad hoc wireless networks. The global positioning system (GPS) has been applied to location-aided services in ad hoc networks [7-9]. This technology has some limitations, as GPS receivers require a clear view of the sky in the lineof-sight of the satellites, which precludes their use in indoor or RF-shadowed environments. Moreover, the size, energy consumption, and cost of GPS receivers can make them impractical for some types of ad hoc networks or for certain types of nodes in an ad hoc network. In [10], position estimates of the network nodes or terminodes (terminals plus nodes) are obtained by triangulating TOA signals. However, the implementation of this scheme has significant overhead and delay problems due to the need to coordinate network topology information among the mobile nodes.

Other approaches to location tracking using network-based signal measurements require a relatively large number of reference nodes with known coordinates placed throughout the network $[11,12]$. The location tracking scheme proposed in [11] uses angle-of-arrival (AOA) measurements to triangulate the position of nodes in the network. The mobility tracking scheme proposed in [12] is closest in spirit to the scheme proposed in the present paper in that it employs Kalman-filtering based on a dynamic system model of mobility. However, the scheme of [12] relies on the presence of at least three special references nodes with known coordinates for each mobile node. In contrast, our proposed tracking scheme requires the presence of a small number of reference nodes, i.e., at least two reference nodes, in the network. Moreover, the scheme of [12] assumes that the model parameters are known, whereas our scheme re-estimates the model parameters at each estimation cycle.

The work described in the present paper extends the AR-1 model-based mobility tracking scheme developed for cellular networks in [1] and the linear system model-based tracking scheme for ad hoc networks in [5]. Unlike cellular networks, ad hoc networks do not have fixed base stations with known coordinates which the mobile units can use as reference points. The relay points in an ad hoc network are generally themselves mobile and their coordinates must also be estimated. Thus, the problem of accurate mobility estimation is more challenging in an ad hoc network. The mobility tracking scheme presented here effectively deals with the constraints imposed by the ad hoc networking environment. The algorithm proposed in the present paper differs from the one presented in our earlier paper [5] in the following aspects: 1) the mobility model used is the AR-1 model, which supports adaptive re-estimation of model parameters, rather than the linear system model; 2) the Kalman filter-based state estimation is distributed among the nodes.

The remainder of the paper is organized as follows. Section 2 reviews the linear system model of mobility as well as the AR-1 mobility model of [1]. Section 
3 presents our distributed mobility tracking algorithm. Some representative numerical results are discussed in section 4 . Finally, section 5 concludes the paper.

\section{Mobility Models}

In this section, we briefly describe two mobility models: the linear system model (cf. [4]) and the AR-1 mobility model introduced in [1]. The AR-1 model provides the basis for our proposed mobility tracking scheme as discussed in section 3 . The linear system model is used to generate mobility patterns for our simulation experiments presented in section 4 .

\subsection{Linear System Mobility Model}

The linear system model has been applied to tracking dynamic targets in [13, $14]$ and location tracking in cellular networks $[4,15,16]$. The mobile unit's state at time $n$ is defined by a (column) vector ${ }^{1}$

$$
\boldsymbol{s}_{n, i}=\left[x_{n, i}, \dot{x}_{n, i}, \ddot{x}_{n, i}, y_{n, i}, \dot{y}_{n, i}, \ddot{y}_{n, i}\right]^{\prime},
$$

where $x_{n, i}$ and $y_{n, i}$ specify the position, $\dot{x}_{n, i}$ and $\dot{y}_{n, i}$ specify the velocity, and $\ddot{x}_{n, i}$ and $\ddot{y}_{n, i}$ specify the acceleration of the mobile node $N_{i}$ at time $n$ in the $x$ and $y$ directions in a two-dimensional grid. The discrete-time state equation of the linear dynamic system is given by

$$
\boldsymbol{s}_{n+1, i}=A \boldsymbol{s}_{n, i}+B \boldsymbol{u}_{n}+\boldsymbol{w}_{n},
$$

where $\boldsymbol{u}_{n}=\left[u_{x, n}, u_{y, n}\right]^{\prime}$ is a vector of two independent semi-Markov processes and the process $\boldsymbol{w}_{n}$ is a $6 \times 1$ discrete-time zero mean, stationary Gaussian vector with autocorrelation function $R_{w}(k)=\delta_{k} Q$, where $\delta_{0}=1$ and $\delta_{k}=0$ when $k \neq 0$, and $Q$ is the covariance matrix of $\boldsymbol{w}_{n}$. The matrices $A, B$, and $Q$ depend on the sampling time interval $T$ and another parameter $\alpha$, which is defined as the reciprocal of the acceleration time constant (see [15]).

The specification of an optimal set of linear system model parameters, i.e., $\alpha$ and the semi-Markov processes $u_{x, n}$ and $u_{y, n}$ is not straightforward in general. Mobility tracking schemes derived from the linear dynamic system model are accurate as long as the model parameters match the mobility characteristics of the user. However, they cannot adapt to significant changes in the model parameters over time.

\section{$2.2 \quad$ AR-1 Mobility Model}

The AR-1 mobility model differs from the linear system model in that the semiMarkov processes, i.e., $u_{x, n}$ and $u_{y, n}$, are not incorporated in state evolution. In the AR-1 mobility model [1], the mobility state of node $N_{i}$ at time $n$ is also

\footnotetext{
$\overline{1}$ The notation' indicates the matrix transpose operator.
} 
defined by (1). The AR-1 model for the mobility state $\boldsymbol{s}_{n, i}$ of node $N_{i}$ is given as follows:

$$
\boldsymbol{s}_{n+1, i}=A_{i} \boldsymbol{s}_{n, i}+\boldsymbol{w}_{n, i},
$$

where $A_{i}$ is a $6 \times 6$ transformation matrix, the vector $\boldsymbol{w}_{n, i}$ is a $6 \times 1$ discrete-time zero mean, white Gaussian process with auto-covariance matrix $Q_{i}$. Numerical experiments with the real mobility data of mobile stations (cf. [1]) indicate that the AR-1 model is sufficient to describe the movement dynamics of nodes in a mobile networking environment. In the AR-1 model, the matrix $A_{i}$ and the covariance matrix $Q_{i}$ are completely general and can be estimated based on training data using the Yule-Walker equations [6]. This allows the model to accurately characterize a wide class of mobility patterns. Using the Yule-Walker equations (see [1]), a MMSE (Minimum Mean Squared Error) estimate of $A_{i}$, denoted by $\hat{A}_{i}^{(n)}$, where $n$ specifies the amount of training data available, can be obtained from the mobility state data $\boldsymbol{s}_{1, i}, \cdots, \boldsymbol{s}_{n, i}$. Similarly, an estimate, $\hat{Q}_{i}^{(n)}$, of the noise covariance matrix can be obtained.

\section{Mobility Tracking Algorithm}

In our proposed distributed mobility tracking scheme, each node tracks its own mobility state using the signal strengths derived from packets received from neighbor nodes. Each node periodically broadcasts its predicted mobility state, i.e., $\hat{s}_{n \mid n-1, j}$ and associated covariance, $M_{n \mid n-1, j}$, to its neighbors. The overhead can be further reduced if only the state and covariance information related to position coordinates are transmitted, as the node estimating its mobility state does not need the full state and covariance matrices. This position information is either piggybacked onto data packets if the data communication is already in process between the nodes or written into special HELLO packets when there is no data to transmit. Thus, the communication overhead incurred by the distributed scheme is relatively small and the computational overhead of the state estimation process is distributed among the individual nodes.

The given node $N_{1}$ requires partial knowledge of $\hat{\boldsymbol{s}}_{n \mid n-1, j}$ and $M_{n \mid n-1, j}$ from its neighbor nodes $N_{j}, j=0,2,3$, in addition to the RSSI or TOA signal measurements. We remark that not all elements of $\hat{\boldsymbol{s}}_{n \mid n-1, j}$ and $M_{n \mid n-1, j}$ are used in the mobility state estimation. The HELLO packets, transmitted once every estimation cycle, contain the position estimates of $N_{j}$, i.e., the first and fourth elements of the estimated mobility state vector $\left(\hat{\boldsymbol{s}}_{n \mid n-1, j}(1)\right.$ and $\left.\hat{\boldsymbol{s}}_{n \mid n-1, j}(4)\right)$ and their associated variances, i.e., $M_{n \mid n-1, j}(1,1)$ and $M_{n \mid n-1, j}(4,4)$, where the numbers in parentheses indicate indices of the matrix.

Fig. 1 shows a block diagram of the main components of the mobility tracking algorithm to be executed at each node, in this case node $N_{1}$. The observation vector $\boldsymbol{o}_{n, 1}$ is provided as input to a pre-filter module. The output of the prefilter, $\hat{\boldsymbol{o}}_{n, 1}$ is provided as input to both the initialization module and the Kalman filter. The initialization module initializes the node's positions in a local coordinate system as described in [10]. The initial mobility state, i.e., $\hat{\boldsymbol{s}}_{0,1}$, consists of 


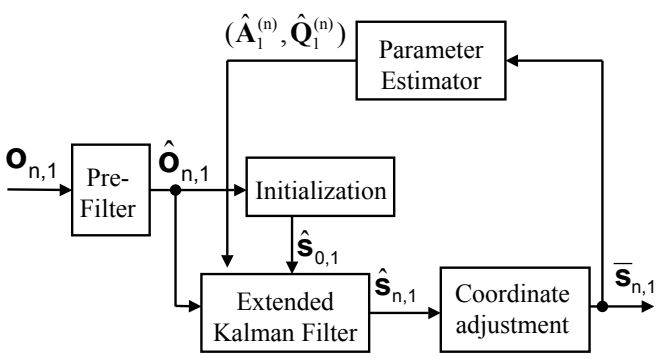

Fig. 1. Block diagram of mobility tracking algorithm.

the initial position coordinates with the velocity and acceleration components set to zero. The output $\hat{\boldsymbol{s}}_{n, 1}$ of the Kalman filter is the estimate of the mobility state in the local coordinate system. Finally, the coordinate adjustment module transforms the local state vector $\hat{\boldsymbol{s}}_{n, 1}$ into a global state vector $\overline{\boldsymbol{s}}_{n, 1}$. Coordinate adjustment can be done using the scheme of [10], which uses standard techniques of rotation and translation of cartesian coordinate systems. The state estimates $\hat{\boldsymbol{s}}_{n, i}$ are used to re-estimate the AR-1 model parameters $\hat{A}_{i}^{(n)}$ and $\hat{Q}_{i}^{(n)}$ at time $n$. A recursive model parameter estimator for the AR-1 model is given in [1]. Prefiltering is done to reduce the noise in the observation data provided as input to the estimation scheme. The pre-filter consists of an averaging filter and outputs a vector of reduced-noise observations $\hat{\boldsymbol{o}}_{n, 1}$ (cf. [16]).

\subsection{Observation vector}

We assume that a given node $N_{1}$ receives RSSI or TOA signals from three or more neighboring mobile nodes. Observability arguments (cf. [16]) show that Kalman filter-based estimation can yield meaningful state estimates with fewer than three observations from fixed base stations with known locations, but tracking accuracy improves with the number of independent observations. For node $N_{1}$, an observation vector is constructed consisting of the three signal measurements from neighbor nodes, i.e.,

$$
\boldsymbol{o}_{n, 1}= \begin{cases}\left(p_{n, 10}, p_{n, 12}, p_{n, 13}\right)^{\prime}, & \text { for RSSI, } \\ \left(\tau_{n, 10}, \tau_{n, 12}, \tau_{n, 13}\right)^{\prime}, & \text { for TOA }\end{cases}
$$

where $p_{n, i j}$ denotes the RSSI received at node $N_{i}$ from node $N_{j}$ and $\tau_{n, i j}$ denotes the TOA measured at $N_{i}$ from $N_{j}$. The general observation or measurement equation in a wireless environment is written as follows:

$$
\boldsymbol{o}_{n, i j}=h\left(\Delta \boldsymbol{s}_{n, i j}\right)+\rho_{n, i j},
$$


where $h(\cdot)$ is a nonlinear function that relates the state $\boldsymbol{s}_{n, i j}$ to the observation data (either RSSI or TOA) and $\rho_{n, i j}$ is a zero mean, stationary Gaussian process (cf. [1]).

To apply the extended Kalman filter for estimating the state vector, the observation $o_{n, i j}$ can be linearized about the estimated state vectors ${ }^{2} \hat{\boldsymbol{s}}_{n \mid n-1, i}$ and $\hat{\boldsymbol{s}}_{n \mid n-1, j}(\mathrm{cf} .[1])$ :

$$
o_{n, i j} \approx h\left(\Delta s_{n, i j}^{*}\right)+H_{n, i j}\left(\Delta s_{n, i j}-\Delta s_{n, i j}^{*}\right)+\rho_{n, i j},
$$

where $\Delta \boldsymbol{s}_{n, i j}^{*}=\hat{\boldsymbol{s}}_{n \mid n-1, i}-\hat{\boldsymbol{s}}_{n \mid n-1, j}$ and the vector $H_{n, i j}$ is given by (cf. [1])

$$
H_{n, i j}=\frac{\partial h}{\partial \Delta s} \mid \Delta s=\Delta \boldsymbol{s}_{n, i j}^{*} .
$$

Define $\zeta_{n, i j}=H_{n, i j}\left(\boldsymbol{s}_{n, j}-\hat{\boldsymbol{s}}_{n \mid n-1, j}\right)$. The conditional density of $\zeta_{n, i j}$ given the previous observations can be approximated by a Gaussian density as follows:

$$
f\left(\zeta_{n, i j} \mid \boldsymbol{O}_{n, j}\right) \sim N\left(0, H_{n, i j} M_{n \mid n-1, j} H_{n, i j}^{\prime}\right),
$$

where $M_{n \mid n-1, j}=\operatorname{Cov}\left[\boldsymbol{s}_{n, j} \mid \boldsymbol{O}_{n-1, j}\right]$. The above expression uses the fact that the conditional density of a mobility state given the observations can be approximated by a Gaussian density (cf. [17]), i.e., $f\left(\boldsymbol{s}_{n, j} \mid \boldsymbol{O}_{n, j}\right) \sim N\left(\hat{\boldsymbol{s}}_{n \mid n, j}, M_{n \mid n, j}\right)$. The expression for the conditional density can be verified easily using (3) and (6). The linearized observation vector for node $N_{1}$ is then given as

$$
\boldsymbol{o}_{n, 1} \approx h\left(\Delta \boldsymbol{s}_{n, 1}^{*}\right)+H_{n, 1}\left(\boldsymbol{s}_{n, 1}-\hat{\boldsymbol{s}}_{n \mid n-1,1}\right)+\boldsymbol{\zeta}_{n, 1}+\boldsymbol{\rho}_{n, 1},
$$

where

$$
\begin{array}{ll}
h\left(\hat{\boldsymbol{s}}_{n, 1}\right)=\left[h\left(\Delta \hat{\boldsymbol{s}}_{n, 10}\right), h\left(\Delta \hat{\boldsymbol{s}}_{n, 12}\right), h\left(\Delta \hat{\boldsymbol{s}}_{n, 13}\right)\right]^{\prime}, & H_{n, 1}=\left[H_{n, 10}^{\prime}, H_{n, 12}^{\prime}, H_{n, 13}^{\prime}\right]^{\prime}, \\
\boldsymbol{\zeta}_{n, 1}=\left[\zeta_{n, 10}, \zeta_{n, 12}, \zeta_{n, 13}\right]^{\prime}, & \boldsymbol{\rho}_{n, 1}=\left[\rho_{n, 10}, \rho_{n, 12}, \rho_{n, 13}\right]^{\prime} .
\end{array}
$$

The noise terms $\zeta_{n, 1 j}$ and $\rho_{n, 1 j}$ are zero-mean independent Gaussian distributed variables with variances $\sigma_{\zeta, 1 j}^{2}$ and $\sigma_{\rho}^{2}$, respectively.

\subsection{Extended Kalman Filter}

The steps in the extended Kalman filter are given as follows (cf. [18]). The algorithm is executed on node $N_{1}$ and $N_{0}, N_{2}$, and $N_{3}$ are the neighbor nodes.

\section{Initialization:}

1. $\hat{\boldsymbol{s}}_{0 \mid-1,1}$ is initialized as discussed in section 3.3

2. $M_{0 \mid-1,1}=I_{6}$

Recursive estimation (time $n, n=1,2, \cdots)$ :

\footnotetext{
${ }^{2}$ We use the Kalman filter notation $\hat{\boldsymbol{s}}_{n \mid n, i}$ and $\hat{\boldsymbol{s}}_{n \mid n-1, i}$ to denote the state estimates for $N_{i}$ at time $n$ given the observations vectors up to times $n$ and $n-1$, respectively.
} 
1. $H_{n, 1 j}=\left.\frac{\partial h}{\partial \Delta \boldsymbol{s}}\right|_{\Delta s=\hat{\boldsymbol{s}}_{n \mid n-1,1}-\hat{\boldsymbol{s}}_{n \mid n-1, j}} ; j=0,2,3$

2. $\sigma_{\zeta, 1 j}^{2}=H_{n, 1 j} M_{n \mid n-1, j} H_{n, 1 j}^{\prime} ; j=0,2,3$

3. $R_{1}=\operatorname{diag}\left[\sigma_{\zeta, 10}^{2}, \sigma_{\zeta, 12}^{2}, \sigma_{\zeta, 13}^{2}\right]+\sigma_{\rho}^{2} * I_{3}$

4. $K_{n, 1}=M_{n \mid n-1,1} H_{n, 1}^{\prime}\left(H_{n, 1} M_{n \mid n-1,1} H_{n, 1}^{\prime}+R_{1}\right)^{-1}$

5. $\hat{\boldsymbol{s}}_{n \mid n, 1}=\hat{\boldsymbol{s}}_{n \mid n-1,1}+K_{n, 1}\left(\hat{\boldsymbol{o}}_{n, 1}-h\left(\hat{\boldsymbol{s}}_{n \mid n-1,1}\right)\right)$ [Correction step]

6. $\hat{\boldsymbol{s}}_{n+1 \mid n, 1}=A_{1} \hat{\boldsymbol{s}}_{n \mid n, 1}$ [Prediction step]

7. $M_{n \mid n, 1}=\left(I-K_{n, 1} H_{n, 1}\right) M_{n \mid n-1,1}\left(I-K_{n, 1} H_{n, 1}\right)^{\prime}-K_{n, 1} R_{1} K_{n, 1}^{\prime}$

8. $M_{n+1 \mid n, 1}=A_{1} M_{n \mid n, 1} A_{1}^{\prime}+Q_{1}$

where $M_{l \mid k, 1}=\operatorname{Cov}\left(s_{l \mid k, 1}\right), l=n, k \in\{n, n-1\}, K_{n, 1}$ is the Kalman gain matrix, and $R_{1}$ is the covariance matrix of the noise in measurements.

\subsection{Initialization Module}

To create a coordinate system in the network, one of the nodes, say $N_{0}$, assumes the role of the origin. The origin node could be determined by a simple election protocol. The origin node $N_{0}$ then sets its position coordinates as $\left(x_{0}, y_{0}\right)=(0,0)$ and chooses two of its neighbors, say nodes $N_{1}$ and $N_{2}$, respectively, to determine the $x$ and $y$ axes of the local coordinate system. More precisely, $N_{1}$ is chosen to lie on the positive $x$ axis and the coordinates of node $N_{2}$ are chosen to have a positive y-component. The coordinates of $N_{1}$ and $N_{2}$ are initialized as follows (cf. [10]):

$$
\left(x_{1}, y_{1}\right)=\left(\hat{d}_{01}, 0\right), \quad\left(x_{2}, y_{2}\right)=\left(\hat{d}_{02} \cos \theta_{12}, \hat{d}_{1,02} \sin \theta_{12}\right),
$$

where $\hat{d}_{i j}=e^{\left(\kappa_{j}-\hat{o}_{n, i j}\right) / 10 \gamma}$ denotes the initial distance estimate between nodes $N_{i}$ and $N_{j}$, and $\theta_{12}=\arccos \left(\left(\hat{d}_{01}^{2}+\hat{d}_{02}^{2}-\hat{d}_{12}^{2}\right) / 2 \hat{d}_{01} \hat{d}_{02}\right)$ denotes the angle from the position vector corresponding to node $N_{1}$ to that corresponding to $N_{2}$, with $0 \leq \theta_{12} \leq \pi$. The coordinates $\left(x_{1}, y_{1}\right)$ and $\left(x_{2}, y_{2}\right)$ could be computed by the origin node $N_{0}$ and transmitted to nodes $N_{1}$ and $N_{2}$, respectively. After this is done, the nodes $N_{0}, N_{1}$, and $N_{2}$, completely determine a local coordinate system. Once the coordinates of a given node have been initialized, the node broadcasts its coordinates within its neighborhood. If a given node $N_{k}$ has at least three neighbors, $N_{i}, N_{j}, N_{l}$, with their coordinates already defined in the local coordinate system (e.g., $N_{0}, N_{1}$, and $N_{2}$ ), the node can initialize its position coordinates via triangulation as follows:

$$
\left[\begin{array}{l}
x_{k} \\
y_{k}
\end{array}\right]=0.5\left[\begin{array}{cc}
x_{i}-x_{j} & y_{i}-y_{j} \\
x_{i}-x_{l} & y_{i}-y_{l}
\end{array}\right]^{-1}\left[\begin{array}{l}
-\hat{d}_{i k}^{2}+\hat{d}_{j k}^{2}+x_{i}^{2}-x_{j}^{2}+y_{i}^{2}-y_{j}^{2} \\
-\hat{d}_{i k}^{2}+\hat{d}_{l k}^{2}+x_{i}^{2}-x_{l}^{2}+y_{i}^{2}-y_{l}^{2}
\end{array}\right] .
$$

The initialization process starts at $N_{0}$, i.e., the origin of the local coordinate system and propagates outward until a maximal set of nodes in the network is initialized within the coordinate system. 


\section{Numerical Results}

In this section, we present some representative simulation results to demonstrate the operation and performance of the proposed mobility tracking scheme.

\subsection{Simulation setup and assumptions}

The results presented here were obtained using a Matlab-based simulation model of an ad hoc network comprised of 30 nodes. Random mobile trajectories for each of the nodes were generated using the linear system model discussed in section 2.1. The first 50 position coordinates of each node are used to initialize the AR-1 model parameters as described in section 2.2. An appropriate training data set is required for proper initialization of the AR-1 model parameters. In our simulation experiments, each mobile trajectory contains almost 350 sample points and the transmission range of all the nodes is assumed to be $400 \mathrm{~m}$. Each node uses signal measurements from all neighbor nodes to estimate its mobility state.

RSSI measurements at time $n$ were generated using the lognormal shadowing fading model [19]:

$$
p_{n, i j}=\kappa_{j}-10 \gamma \log \left(d_{n, i j}\right)+\psi_{n, i j}
$$

where $p_{n, i j}$ is the RSSI received at node $N_{i}$ from node $N_{j}, d_{n, i j}$ is the distance between the nodes, and $\psi_{n, i j}$ is a zero mean, stationary Gaussian process with a standard deviation of $4 \mathrm{~dB}$. The parameter $\kappa_{j}$ is assumed to be zero and $\gamma$ is set to 8. TOA measurements yield similar results to RSSI measurements (cf. [1]). The difference in performance in real scenarios depends on the accuracy with which the signal measurements are collected, especially in the case of TOA, and on the accuracy of the assumed lognormal signal propagation model in the case of RSSI.

The initial positions of these nodes are assumed to be uniformly distributed in a square $600 \mathrm{~m} \times 600 \mathrm{~m}$ area, but they are subsequently allowed to move outside this area. The initial speed and acceleration, in each dimension, are assumed to be zero for all nodes. The discrete-time interval is set as $T=0.1 \mathrm{~s}$. The dynamic system model parameters are set as follows: $\alpha=1 \mathrm{~s}^{-1}$ and $\sigma_{1}=1 \mathrm{~dB}$ (cf. section 2.1). The discrete command processes $u_{x}(t)$ and $u_{y}(t)$ for the three mobile nodes are independent semi-Markov processes, each taking on five possible levels of acceleration comprising the set $\mathcal{L}=\{-1,-0.5,0,0.5,1\}$ in units of $\mathrm{m} / \mathrm{s}^{2}$. This set of acceleration levels is capable of generating a wide range of dynamic motion. The initial probability vector $\boldsymbol{\pi}$ for the semi-Markov model (SMM) governing $u_{x}(t)$ and $u_{y}(t)$ is initialized to a uniform distribution. The elements of the transition probability matrix for the SMM are initialized to a common value of $1 / 5$. We assume that the dwell times in each state are uniformly distributed with a common mean value of $2 T \mathrm{~s}$, where $T$ is the sampling interval. 


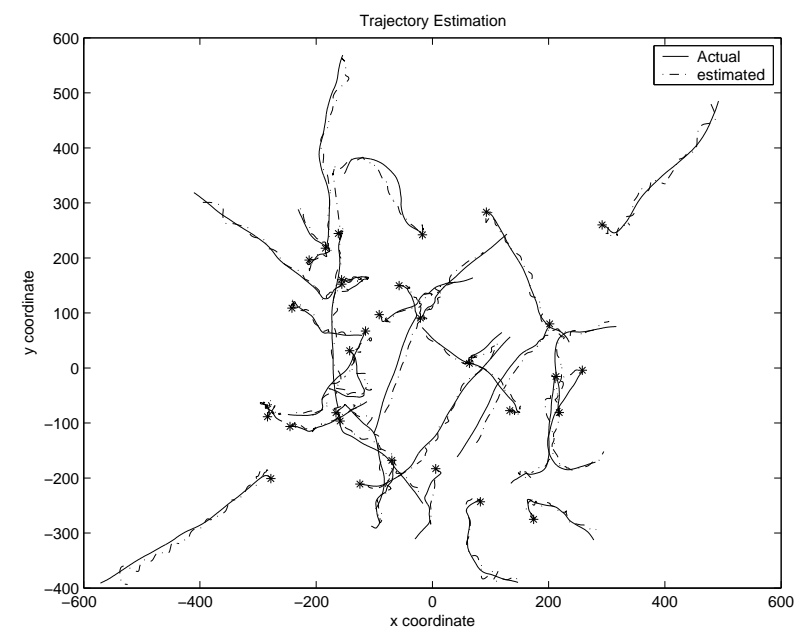

Fig. 2. Mobility tracking with two reference nodes.

\subsection{Mobility estimation}

A sample simulation result showing the tracking of 30 mobile nodes is illustrated in Fig. 2, where the coordinates are represented in meters. The initial coordinates of each node are marked with $*$, as shown in Fig. 2. The first 50 position coordinates of each node are used to initialize the AR-1 model parameters $A_{i}$ and $Q_{i}$ for each node $N_{i}$. Fig. 2 shows mobility tracking results when two additional stationary reference nodes are located at $(0,-300)$ and $(0,300)$ (not shown in Fig. 2). One node is selected to serve as the origin of the local coordinate system and the two nodes closest to the origin are chosen to determine the $x$ and $y$ axes of the local system. We note that the estimation error is larger for the nodes moving further away from the rest of the nodes than the nodes which stay closer to the other nodes. As nodes move further apart, there are fewer neighbor nodes to provide independent observations. We also remark that tracking accuracy generally improves with increasing nodal density, again since the presence of more neighbors provides more observation data for the state estimation process.

We compared the performance of the proposed tracking scheme with a similar tracking scheme based on the linear system model, in which the model parameters are assumed to be known. Mobility tracking performance can be quantified in terms of root mean square estimation error (RMSE). We use root mean squared error (RMSE) as a figure of merit to compare a given trajectory $\left\{x_{n}, y_{n}\right\}$ and its estimated trajectory $\left\{\hat{x}_{n}, \hat{y}_{n}\right\}$ :

$$
\operatorname{RMSE}=\sqrt{\frac{1}{N} \sum_{n=1}^{N}\left[\left(\hat{x}_{n}-x_{n}\right)^{2}+\left(\hat{y}_{n}-y_{n}\right)^{2}\right]}
$$


Table 1 shows RMSE results for various scenarios in terms of the sample mean, $\mu_{R M S E}$, and standard deviation, $\sigma_{R M S E}$, of the RMSE statistic, which are computed using 500 independently generated sample experiments. The first column in the table indicates the estimation scheme and the second column shows the availability of model parameters. The third column indicates the standard deviation, $\sigma_{\psi}$, of the shadowing noise in the RSSI measurements. The table shows that the integrated AR-1 estimator has a slightly larger mean RMSE compared to the state estimator with complete knowledge of the linear system model parameters. Interestingly, the standard deviation of the RMSE is smaller for the AR-1 based estimator. From Table 1, we also observe that increasing the shadowing nodes tends to degrade tracking performance, but tracking performance remains fairly stable even for a standard deviation of $\sigma_{\psi}=8 \mathrm{~dB}$. Moreover, the presence of three reference nodes in the network improves the accuracy of the estimator when compared against the case of two reference nodes.

\begin{tabular}{lcc|cc}
\hline \hline Estimation Scheme & Model Parameters $\sigma_{\psi}(\mathrm{dB})$ & $\mu_{\mathrm{RMSE}}(\mathrm{m})$ & $\sigma_{\mathrm{RMSE}}(\mathrm{m})$ \\
\hline Linear System & known & 4 & 4.39 & 8.4 \\
Integrated AR-1 with 2 references & unknown & 4 & 8.78 & 3.99 \\
Integrated AR-1 with 3 references & unknown & 4 & 7.38 & 1.46 \\
\hline Linear System & known & 8 & 7.45 & 12.6 \\
Integrated AR-1 with 2 references & unknown & 8 & 13.62 & 5.66 \\
Integrated AR-1 with 3 references & unknown & 8 & 10.96 & 2.91 \\
\hline \hline
\end{tabular}

Table 1. RMSE of mobility tracking over 500 sample experiments.

\section{Conclusion}

We have proposed a distributed scheme for tracking node mobility in ad hoc networks based on an autoregressive model of mobility. The tracking scheme estimates both the current mobility state and re-estimates the mobility model parameters in an integrated fashion. Thus, the scheme does not require prior knowledge of mobility model parameters and can adapt to wireless networking scenarios where the mobility model parameters are not known or may change with time. Simulation results show that the proposed tracking scheme performs accurately under a variety of wireless networking scenarios with a small number of reference nodes. The mobility tracking scheme can be applied in a variety of scenarios to enhance the performance of routing, mobility management, and resource allocation in mobile ad hoc networks. In ongoing work, we are investigating the application of the mobility tracking scheme to predict future link availability in order to enable more efficient routing and improved quality-ofservice provisioning in ad hoc networks. 


\section{References}

1. Zaidi, Z.R., Mark, B.L.: Mobility Estimation for Wireless Networks Based on an Autoregressive Model. In: Proc. of IEEE Globecom, Dallas, TX (2004 (to appear))

2. Bettstetter, C.: Smooth is better than sharp: A random mobility model for simulation of wireless networks. In: Proc. of ACM MSWiM. (2001) 19-27

3. Camp, T., Boleng, J., Davies, V.: Survey of mobility models for ad hoc network research. Wireless Communication and Mobile Computing (WCMC), Special issue on Mobile Ad Hoc Networking 2 (2002) 483-502

4. Liu, T., Bahl, P., Chlamtac, I.: Mobility modeling, location tracking, and trajectory prediction in wireless ATM networks. IEEE J. Selected Areas in Comm. 16 (1998) 922-936

5. Zaidi, Z.R., Mark, B.L.: A mobility tracking model for wireless ad hoc networks. In: Proc. of IEEE WCNC. Volume 3., New Orleans, LA (2003) 1790-1795

6. Lim, J.S., Oppenheim, A.V.: Advanced Topics in Signal Processing. Prentice Hall, Englewood Cliffs, NJ 07632 (1987)

7. Basagni, S., Chlamtac, I., Syrotiuk, V.R., Woodward, B.A.: A Distance Routing Effect Algorithm for Mobility (DREAM). In: Proc. of ACM MobiCom. (1998) 76-84

8. Basu, P., Khan, N., Little, T.D.C.: A mobility-based metric for clustering in mobile ad hoc networks. In: Proc. of Distributed Computing System Workshop. (2001) $413-418$

9. Blazevic, L., Buttyan, L., Capkun, S., Giordano, S., Hubaux, J., Boudec, J.: SelfOrganization in Mobile Ad-hoc Networks: The Approach of Terminodes. IEEE Communication Magazine 39 (2001) 166-174

10. Capkun, S., Hamdi, M., Hubaux, J.: GPS-free positioning in mobile ad-hoc networks. In: Proc. of 34th HICSS. (2001) 3481-3490

11. Niculescu, D., Nath, B.: Ad hoc positioning system (APS) using AOA. In: Proc. of IEEE Infocom. (2003) 1734-1743

12. Pathirana, P.N., Savkin, A.V., Jha, S.: Mobility modeling and trajectory prediction for cellular networks with mobile base stations. In: Proc. of ACM MobiHoc 2003. (2003) 213-221

13. Singer, R.A.: Estimating optimal tracking filter performance for manned maneuvering targets. IEEE Trans. Aerosp. Elect. Syst. 6 (1970) 473-483

14. Moose, R.L., Vanlandingham, H.F., McCabe, D.H.: Modeling and estimation for tracking maneuvering targets. IEEE Trans. Aerosp. Elect. Syst. 15 (1979) 448-456

15. Mark, B.L., Zaidi, Z.R.: Robust mobility tracking for cellular networks. In: Proc. of IEEE ICC. Volume 1., New York, NY (2002) 445-449

16. Zaidi, Z.R., Mark, B.L.: Real-time Mobility Tracking Algorithms for Cellular Networks based on Kalman Filtering. IEEE Trans. on Mobile Computing (to appear) (2005)

17. H. Vincent Poor: An Introduction to Signal Detection and Estimation. SpringerVerlag, New York (1988)

18. Brown, R.G., Hwang, P.Y.: Introduction to Random Signals and Applied Kalman Filtering. 3rd edn. John Wiley \& Sons, New York (1997)

19. Gudmundson, M.: Correlation model for shadowing fading in mobile radio systems. Electronic Letters 27 (1991) 2145-2146 